\title{
EFFICIENT PLASMA PRODUCTION IN LOW BACKGROUND NEUTRAL PRESSURES WITH THE M2P2 PROTOTYPE
}

\author{
T. Ziemba", P. Euripides, ${ }^{\dagger}$ R. Winglee ${ }^{\ddagger}$, J. Slough $^{\S}$, L. Giersch ${ }^{* \star}$ \\ University of Washington, Seattle, WA
}

\begin{abstract}
Mini-Magnetospheric Plasma Propulsion (M2P2) seeks the creation of a large-scale (10 $\mathrm{km}$ radius) magnetic wall or bubble (i.e. a magnetosphere) by the electromagnetic inflation of a small-scale $(20 \mathrm{~cm}$ radius) dipole magnet. The inflated magnetosphere will intercept the solar wind and thereby provide high-speed propulsion with modest power and fuel requirements due to the gain provided by the ambient medium. Magnetic field inflation is produced by the injection of plasma onto the dipole magnetic field eliminating the need for large mechanical structures and added material weight at launch. For successful inflation of the magnetic bubble a beta near unity must be achieved along the imposed dipole field. This is dependent on the plasma parameters that can be achieved with a plasma source that provide continuous operation at the desired power levels of 1 to 2 kilowatts. Over the last two years we have been developing a laboratory prototype to demonstrate the inflation of the magnetic field under space-like conditions. In this paper we will present some of the latest results from the prototype development at the University of Washington and show that the prototype can produce high ionization efficiencies while operating in near space like neutral background pressures producing electron temperatures of a few tens of electron volts. This allows for operation with propellant expenditures lower then originally estimated.
\end{abstract}

\section{INTRODUCTION}

The need for cost effective solutions allowing for high mass fractions of payload to propellant for in space propulsion has lead to the development and utilization of plasma-based systems. Plasma thrusters are more efficient then chemical propulsion thus reducing propellant requirements but they suffer from low thrust

\footnotetext{
* Graduate Student

${ }^{\dagger}$ Post Doc

* Professor

$\S$ Assistant Professor

** Graduate Student
}

levels limiting the possible missions that may be undertaken with these thrusters to those of low mass and generally of long durations. The high power efficiencies realized by ion and Hall thrusters are due to the non-thermal plasma produced by an imposed electric field, but this leads to space charge limitations on the plasma density that can be maintained by the device, limiting overall thrust levels. Additionally, anode and cathode wear and sputtering are of concern as they may lead to degraded performance for longer missions.

Other plasma base propulsion concepts take advantage of inductive or wave coupled plasma sources, in particular helicon sources, that have been used widely in laboratory and industrial applications. ${ }^{1,2}$ Helicon sources are able to produce an order in magnitude increase in plasma densities without electrodes contacting the plasma.,4 Unfortunately, most prior laboratory experiments have shown the plasmas produced by these sources to be thermal. ${ }^{5}$ Therefore, additional heating of the plasma ions is required to see efficiencies gains similar to Hall thrusters which adds to the overall power requirements to operate such a system.

The M2P2 concept attempts to couple with the ambient solar wind to provide an overall power efficiency gain $>1$ with thrust levels predicted by simulation of several Newtons. ${ }^{6}$ To accomplish this, plasma must be produced and injected along an imposed dipole magnetic field as has been previously described. ${ }^{\top}$ Plasma parameters required for the M2P2 concept are similar to those produced by helicon plasma sources. The initial prototype design and experimental testing has been accomplished utilizing a helicon.

Helicon sources are typically operated in the laboratory setting by maintaining a constant neutral backfill of a desired level in the testing chamber on the order of several milli-Torr. In a space-based system, steep neutral pressure gradients would be required down stream of the ionization chamber were the plasma is exhausted into space. It is therefore necessary to ensure that helicon sources were able to run in a continuous manner in which neutrals are introduced into the system, efficiently ionized and the plasma is allowed to flow onto the dipole 
field in as close to space like conditions as possible.

This paper discusses recent work at the University of Washington were plasma parameters were monitored as a function of neutral flow into the M2P2 system. The effect of rising neutral pressure through out the entire chamber was also monitored. Results show that the M2P2 helicon source was able to efficiently ionize neutrals producing plasma temperatures and densities at the source similar to those seen in other experiments. Optimum ionization and power efficiencies were found by controlling the neutral inflow into the source region. Plasma temperatures increase in the equatorial region away from the helicon source suggesting addition coupling down stream of the helicon coil. This is confirmed by the use of an electron energy analyzer, which shows a large drift to the electron velocity distribution. Plasma temperatures in the equatorial region are shown to be a strong function of neutral background pressures where plasma temperatures decrease with increasing neutral pressure and plasma density increases.

\section{EXPERIMENTAL ARRANGEMENT AND OPERATION}

The M2P2 prototype is shown in figure 1 and consists of a dipole magnet, and a halfhelical helicon coil, which is secured around a 3 $\mathrm{cm}$ OD quartz tube. The quartz tube serves two purposes, first it allows for direct insertion of neutrals into the source region via a gas feed tube. Secondly, it provides protection for the helicon coil from the plasma so sputtering and coil degradation is not an issue.

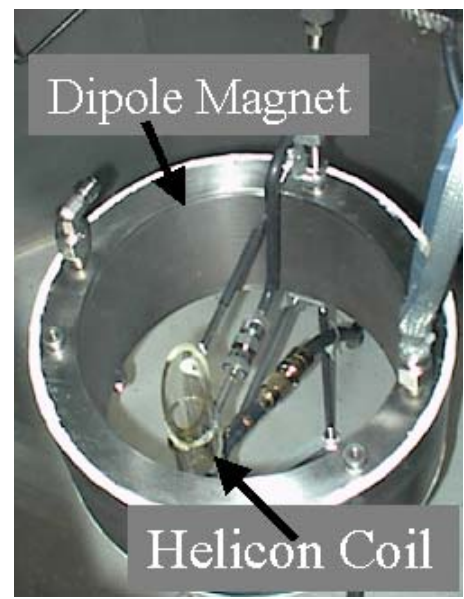

Figure 1, M2P2 Prototype
Figure 2, is a detailed view of the helicon coil and quartz tube. The gas feed can be clearly seen at the center of the tube.

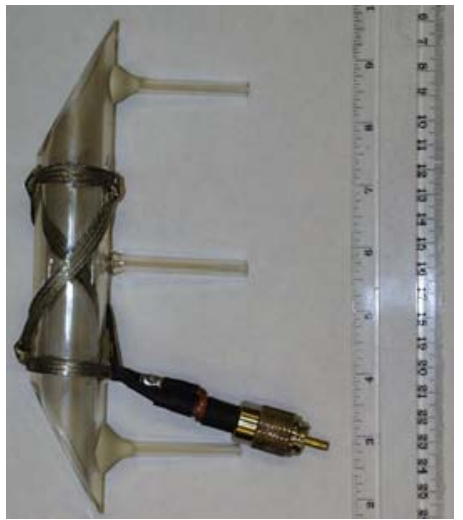

Figure 2, Helicon Source Coil

The coil is connected via an in vacuum $50 \mathrm{Ohm}$ RF cable to a standard L matching network just outside the vacuum chamber. The coil is driven with a Henry Radio 3K Classic amplifier. The amplifier is able to provide a peak power output of 2000 watts. A fast puff valve and a high precision needle valve outside the chamber control neutral gas flow into the source. The experiment and data acquisition is run with Labview $^{\mathrm{TM}}$ software allowing for gas puff parameters to be adjusted on microsecond time scales.

Figure 3 , shows the placement of the prototype in the 4000-liter chamber at the University of Washington test facility. The prototype is hung in the vertical mid-plane and roughly $1 / 3$ of the horizontal distance into the chamber. Electrical and gas feeds are brought to the prototype in one location to minimize the number of magnetic field line that are intersected by any material.

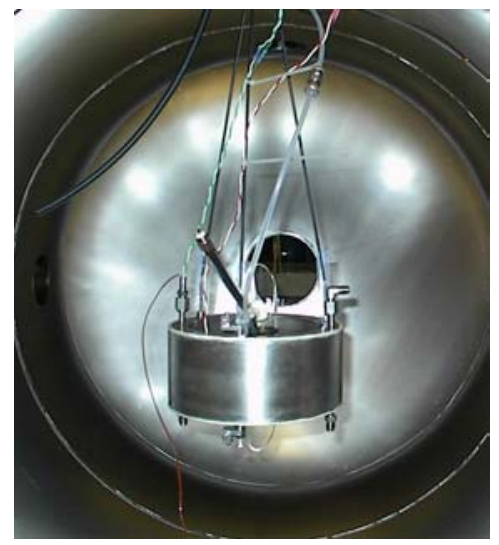

Figure 3, Prototype in 4000-Liter Chamber 
The experiment is run in a pulsed mode with typical plasma shot lengths of one to ten milliseconds. In this manner, plasma parameters could be measured at the source and equatorial region of the dipole prior to build up of significant neutral pressures through out the chamber. A capactive monometer was used to monitor neutral pressures in the chamber and determine neutral flow rates and optimum plasma ignition times. Figure 4 is capactive monometer data for the 4000-liter chamber and shows the point of plasma ignition. The base resolution of monometer is $5 \times 10^{-5}$ Torr. In the cases where backfill neutrals were introduced into the chamber, an ionization gauge was used for determination of pressures below $5 \times 10^{-5}$ Torr.

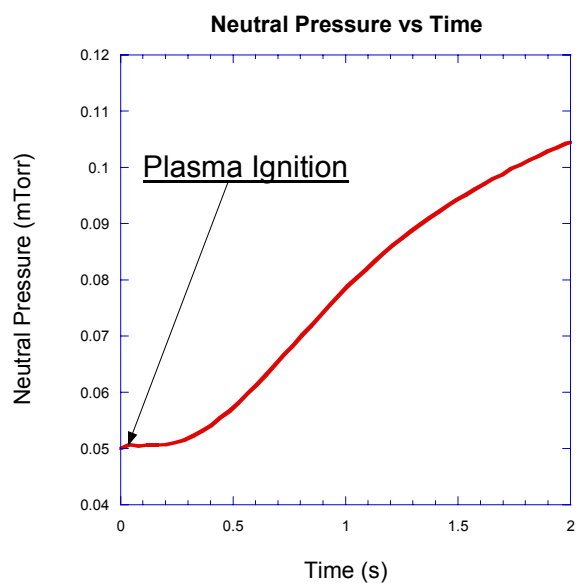

Figure 4, Neutral Pressure vs Time

For typical shots, the plasma ignition was delayed from 20 to $40 \mathrm{~ms}$ after the gas puff. This is roughly the transit time of the gas thru the gas feed tube to reach the helicon source. Figure 3 shows that the ignition occurred at chamber pressures below the minimum detectable level of the capactive manometer. Neutral pressures in the chamber at the time of plasma ignition were calculated to be in the mid $10^{-7}$ Torr. The base pressure for this chamber with current pumping capacity is $1 \times 10^{-7}$ Torr. The ability to run at low neutral pressures allowed for a determination of the plasma characteristics in the most space like conditions possible as well as a characterization of neutral processes which play a key role in this type of discharge.

\section{PLASMA DIAGONSTICS}

To determine plasma parameters a range of plasma diagnostics were used. Generally, the experiment utilized three main diagnostics. These were source and equatorial Langmuir probes, an equatorial electron energy analyzer and a spectrometer for line emission characterization of the source region. Measurements were typically taken at the helicon source region at the top of the dipole magnet and at the equatorial regions in the midplane of the experiment. Figure 5 illustrates the location of probes along with magnetic field strength at probe locations. Magnetic field line mapping from the helicon source region into the equator is also shown. Additionally, the prototype was able to rotate on its axis roughly 30 degrees in each direction. This was done so that azimuthal characteristics of the plasma could be studied.

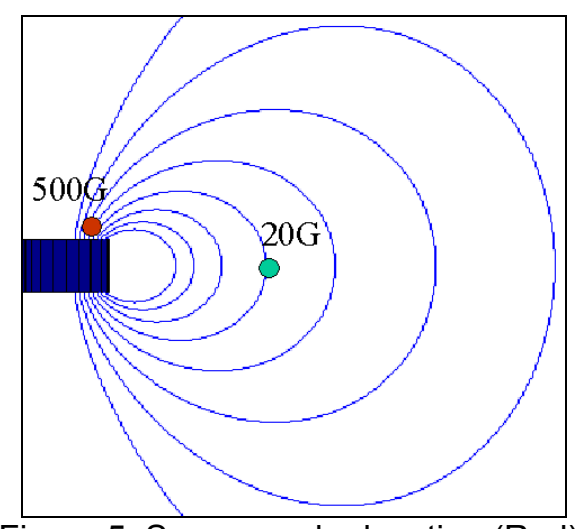

Figure 5, Source probe location (Red), Equatorial probe location (Green)

\section{Source and Radial Langmuir Probes}

Double compensated Langmuir probes in the style commonly used in helicon experiments were located at both the source and equatorial regions. ${ }^{8}$ In each case, probe circuits were of a simple design with each probe biased into ion saturation using batteries or an isolated DC power supply. Current drawn through the probe circuit was measured with a floating oscilloscope. Electron temperatures were measured by changing the probe voltage from shot to shot to produce the characteristic I vs $\mathrm{V}$ curve. Reproducibility from shot to shot is assumed to be good as measured with saturated Langmuir probes. Electron temperature is then calculated by exponential fit 
to the curve. Plasma densities were calculated using the calculated plasma temperature

$$
I_{p} \approx n_{e} \cdot e \cdot A \cdot C_{s}
$$

where, $I_{p}$ is the current in the Langmuir circuit, $A$ is the probe area and $C_{s}$ is the ion sound speed.

Figure 6 shows plasma density vs time for a saturated source probe and I vs $V$ curve with fit for similar shot parameters.
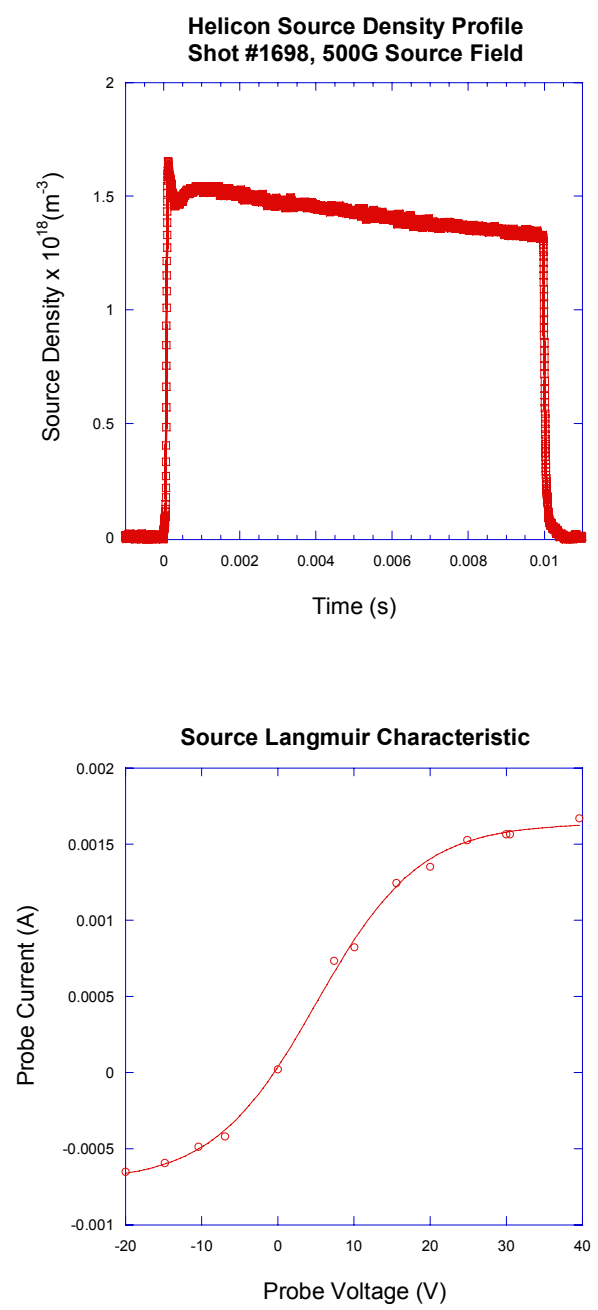

Figure 6, Temporal source density profile (Upper), Langmuir characteristic curve (Lower) with fit found giving $\mathrm{Te}=7 \mathrm{eV}$.

Radial Langmuir data was also collected and processed in a similar manner for both radial temporal profiles and electron temperatures.

\section{Equatorial Electron Energy Analyzer}

An electron energy analyzer was also constructed and placed in the equatorial region of the dipole to measure the electron distribution functions. Similar energy analyzers have been used to measure distribution functions in other helicon experiments with reliable results. ${ }^{9}$ The analyzer consisted of two grids and a collector. Figure 7, shows a schematic of the device and measuring circuit. The ground and retarding grids were made of stainless steel mess with a grid spacing of 1.4 microns and a transparency of 60 percent. The grids and collector plate were isolated from one another with boron nitride washers approximately $1 \mathrm{~mm}$ in thickness.

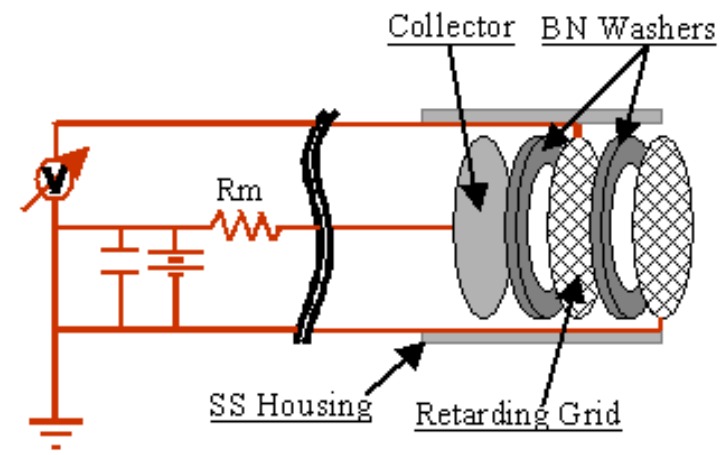

Figure 7, Electron energy analyzer and measuring circuit.

Current collected at the plate was measured as a function of retarding grid voltage allowing determination of the distribution function. The plate battery voltage was 170 volts to ensure collection of all electrons passing thru the retarding grid. Data was collected shot to shot similar to the Langmuir data so that a current vs voltage curve could be developed. Typical data is shown in Figure 8. To determine the electron energy distribution function the data was fit to a $4^{\text {th }}$ or $5^{\text {th }}$ order polynomial and the curve was reconstruction using ten thousand data points. The order of polynomial was determine by the best fit to the data. This is to allow for a more accurate derivative of the current vs voltage curve. This method of curve reconstruction was also employed as noted in reference [9] with good results. However, this method is not optimal as slight changes in the slope of the curve can be removed by the fit, thereby removing the ability to see all the features of the distribution function. 


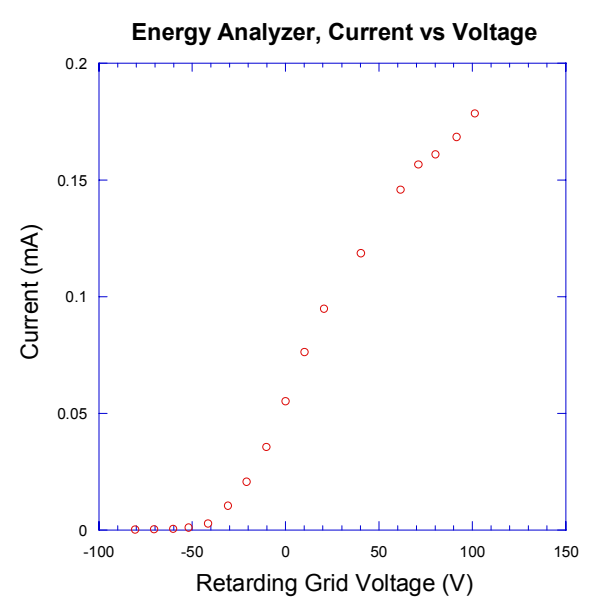

Figure 8, Energy analyzer data

In the future we plan on developing a high frequency and resolution sweeping circuit for the retarding grid voltage. This will enable a direct derivative of the I vs $\mathrm{V}$ curve without fitting. In any case, interesting information about the distribution function can still be seen using the reconstruction method. Figure 9 is a plot of the electron distribution function in the equatorial region obtained using the above mention method.

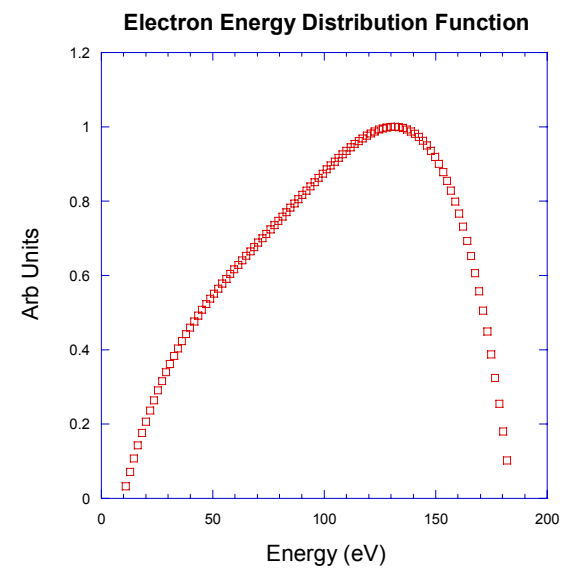

Figure 9, Electron energy distribution.

Distribution is normalized to its peak value. As note that the energy scale has been shifted to account for the sheath voltage developed on the probe.

As evident from Figure 9, even the lowresolution data suggests a non-Maxwellian energy distribution. The peak of the distribution is at roughly $130 \mathrm{eV}$ suggesting that the electron have a non-zero mean velocity. Fitting of the energy distribution to analytic forms for the distribution function that included a non-zero electron drift was conduction and will be discussed in the results section.

\section{RESULTS}

Table 1 details the typical operating and plasma conditions at the source and equatorial locations using argon. Typical shot lengths were from one to ten milliseconds. These conditions are without the addition of neutrals in the equatorial region and with power output maximized at the source region for 500 Gauss source field.

\begin{tabular}{|l|c|c|}
\hline & Source & Equator \\
\hline $\begin{array}{l}\text { Magnetic Field } \\
\text { Strength }(\mathrm{G})\end{array}$ & 500 & $20 \sim 5$ \\
\hline $\begin{array}{l}\text { Helicon Input Power } \\
(\mathrm{W})\end{array}$ & 2000 & NA \\
\hline Plasma Density $\left(\mathrm{m}^{-3}\right)$ & $\sim 2 \times 10^{18}$ & $\sim 1 \times 10^{16}$ \\
\hline $\begin{array}{l}\text { Electron Temperature } \\
(\mathrm{eV})\end{array}$ & $7 \sim 12$ & 25 \\
\hline $\begin{array}{l}\text { Argon Sound Speed } \\
\text { (km/s) }\end{array}$ & 4 & 8 \\
\hline Plasma beta & $8 \times 10^{-3}$ & $.03(20 \mathrm{G})$ \\
\hline Alfen Speed (km/s) & 500 & 300 \\
\hline $\begin{array}{l}\text { Mean Free Path } \\
\text { (MFP) for lonization } \\
\text { (m) }\end{array}$ & $\sim .1$ & $\sim 40000$ \\
\hline MFP Electron-lon & $\sim .5$ & $\sim 20000$ \\
\hline MFP Neutral-lon & $\sim .01$ & $\sim 20000$ \\
\hline
\end{tabular}

Table 1, Standard operational parameters

\section{Source Plasma Output and lonization Efficiencies}

For operation of the prototype in space, the helicon source must be able to run efficiently while limiting gas flow rates to realistic levels. For a clear understanding of dipole confinement and flux inflation it is also necessary to limit neutral pressures in the outer region of the dipole to as near to space like as possible with the current experimental arrangement. This requires that at the helicon source neutral pressures be on the order of several to tens of milli-Torr for effective source operation, without allowing the neutral pressure in the outer region of the dipole to become large. Typical helicon experiments are not operated in this manner, so 
it was necessary to design the experiment to verify operation under these conditions.

It was found that the helicon source was able to operate in a pulsed manner with various flow gas flow rates to the source. Figure 6 above, has shown a plot the source plasma density temporal profile. There is an initial peak in the plasma density for roughly 200 microseconds as source neutrals are consumed, with almost constant density output for the duration of the shot. Operation as a pulsed experiment with precise control of gas flow rates enabled determine of helicon source characteristic as a function of flow rate.

Figure 11 details helicon source density as a function of input flow rate.

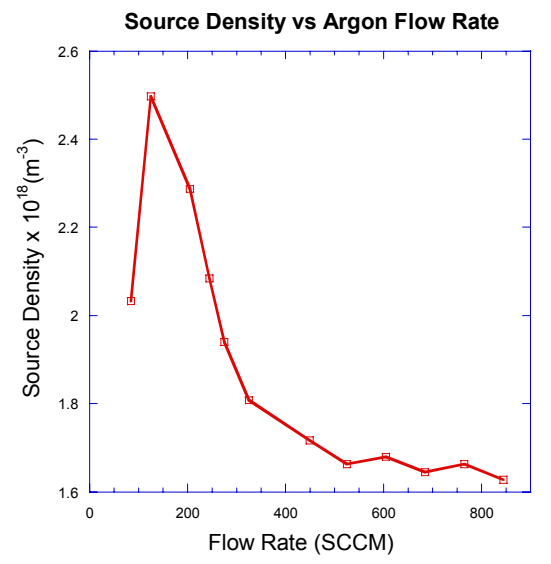

Figure 11, Source density vs Argon flow rates

Density increases to a peak value in source as the flow rate approaches $125 \mathrm{sccm}$, then decrease rapidly. Source temperatures were found to remain approximately in the 7 to $10 \mathrm{eV}$ range for all flow rates. Since the density is only weakly dependent on temperature, the peak corresponds to the maximum source power output. Data in Figure 11 implies that below $125 \mathrm{sccm}$, available power from the source is coupled into ionization. As flow rate is increased beyond $125 \mathrm{sccm}$, maximum power coupling has been reached and additional source neutrals enhance plasma loss via charge exchange, lowering the density output. This can be seen in Figure 12, which shows plots of ionization efficiency vs flow rate with and without wall losses. It is seen that maximum ionization efficiency is at the lowest possible flow rate and decreases with increasing flow rate. When the source is operated in the most efficient mode for ionization, greater that $40 \%$ of the input neutral gas is ionized to plasma, which then flows out of the source and into the dipole equatorial region. When plasma loss to the quartz wall is considered, almost $100 \%$ of the input neutral gas is initially ionized.
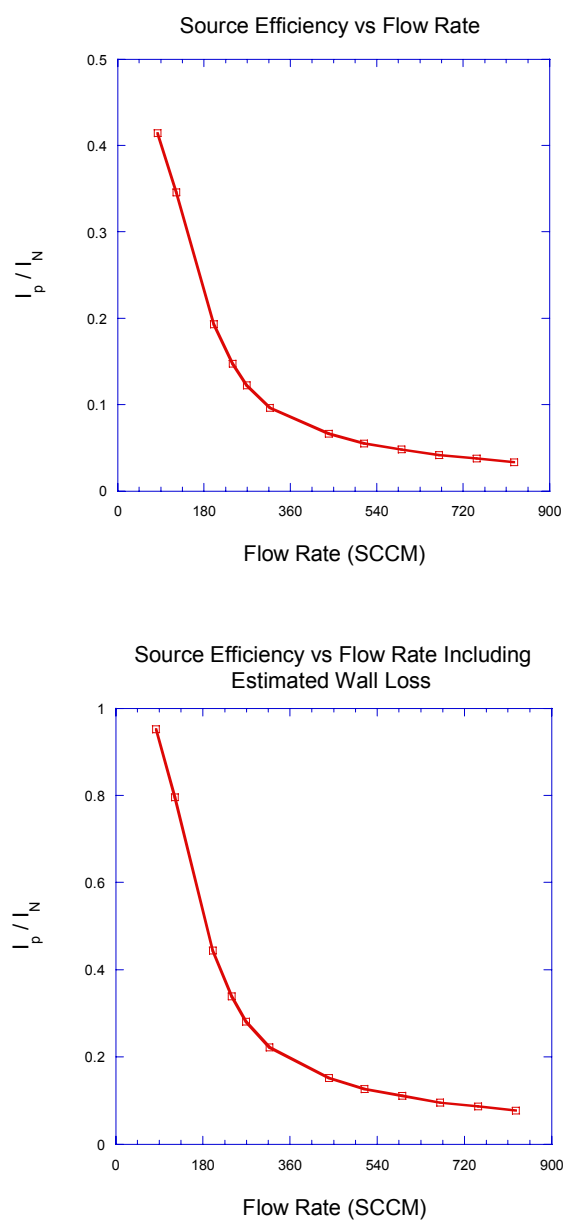

Figure 12, Source ionization efficiencies with and without calculated wall effects.

The above data indicates that the helicon source has high ionization efficiencies when operated in the correct manner. The data also suggests that higher densities at the same ionization efficiencies are possible with increased power levels. Figure 13 shows helicon source density vs power levels. Densities continue to rise with increased input power for fixed input shot parameters. By increasing source power for corresponding flow levels could allow for increased densities while maintaining high ionization efficiencies. This 
would allow for increased plasma betas in the source and equatorial regions.

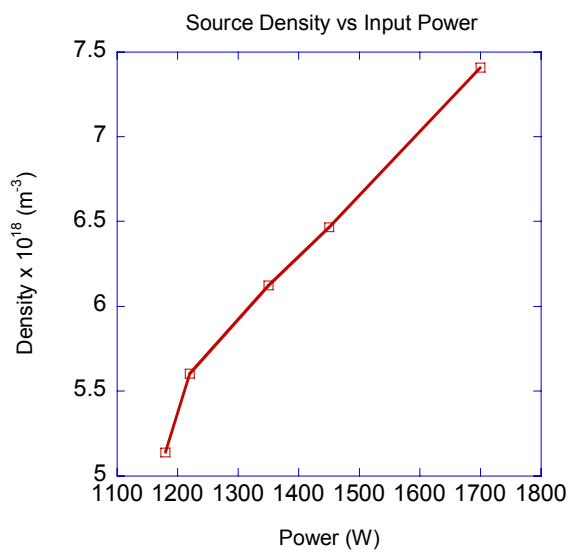

Figure 13, helicon source density vs input power. Overall density levels at the source are 2-3 times larger that previous shows densities due to use of high magnetic field for this study.

\section{Equatorial Plasma Langmuir Profiles and Neutral Dependencies}

Plasma profiles for equatorial region of the dipole were studied with and without the addition of a neutral backfill into the chamber. This was conducted to determine the down stream effect of neutrals away from the helicon coil were peak power coupling is typically see in other helicon experiments. Figure 14 is the temporal current profile of the equatorial Langmuir probe.

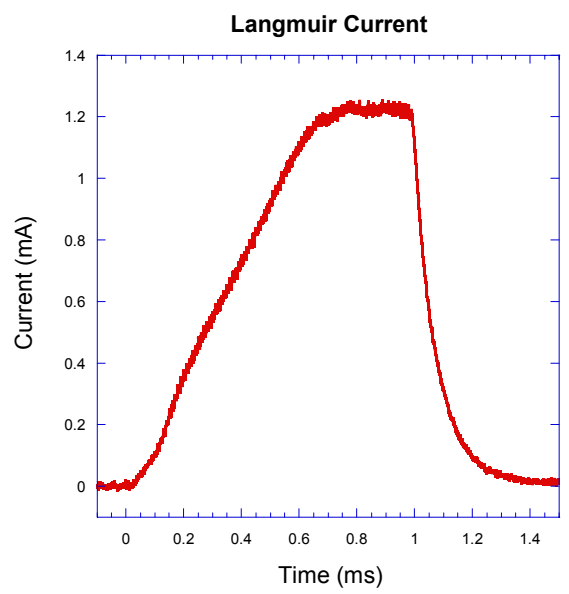

Figure 14, Equatorial Langmuir currnt.

Density is seen to grow as plasma fills in the dipole and reaches a maximum value in 600 microseconds. At this point plasma inflow is matched by plasma loss and density becomes constant.

Figure 15 is the equatorial electron temperature temporal profile for a $4 \mathrm{~ms}$ shot length. The electron temperature is $25 \mathrm{eV}$ and remains constant during the shot. This result supports the theory that more energy is coupled into the plasma down stream from the helicon coil. This turns out to be beneficial effect. The major loss channels are reduced in this region, since there are no boundaries and the neutral density is low.

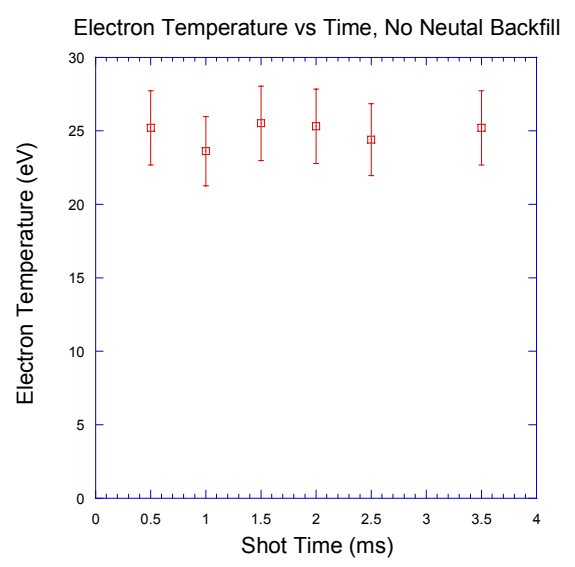

Figure 15, Equatorial electron temperature profile

To determine the effect of neutrals in the equatorial region, the chamber was backfilled with argon gas prior to each plasma shot. The backfill was controlled and monitored with an ionization gauge to ensure the same neutral pressure for each series of data. Figure 16 is the compilation of that data and contains a plot of electron temperature and density as a function of neutral backfill pressure. Data was collected over five orders of magnitude in pressure. The electron temperature is found to decrease in a linear fashion with each decade increase in pressure, ending in a value just below $10 \mathrm{eV}$ at a 1 milli-Torr backfill pressure. Most helicon experiments operate with a backfill of several milli-Torr to several tens of milli-Torr and report electrons temperatures in the $5 \mathrm{eV}$ range. Even more interesting, is the plasma density as a function of fill pressure. The density initially begins to decrease with increasing pressure, but rises sharply at $10^{-4}$ Torr. At this pressure, the mfp for ionization is now decreasing to scale lengths on order of tens 
of meters, falling to just over .5 meters at 1 milliTorr. The increased density is due to secondary plasma production by the ionization of neutrals in the equatorial region. This is a large energy sink for the plasma as ionization reoccurs using electrons generated at the source. This energy loss coupled with increasing losses due to charge exchange reduces the temperature in the region.

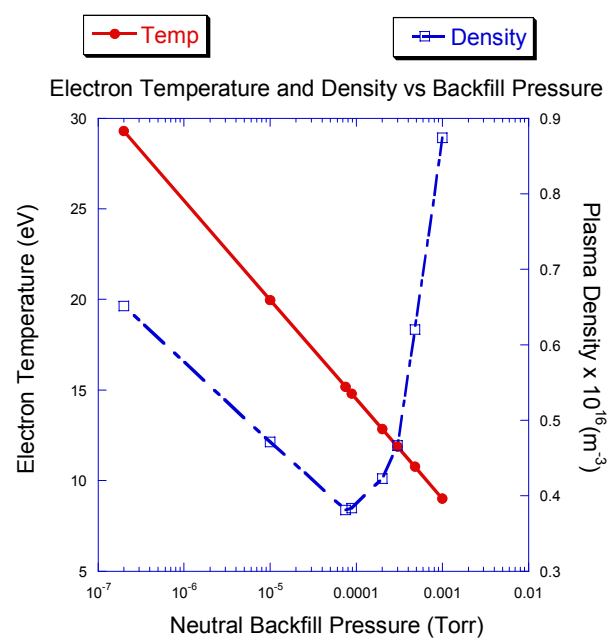

Figure 16, Electron temperatures and densities vs neutral backfill pressure in the equatorial region

From Figure 16, it is seen that if neutral pressures downstream of the source rise to levels above $10^{-4}$ Torr, space like conditions are violated and neutral interactions in this region will dominate.

Further energy coupling to neutrals in the equatorial region is also confirmed from radial Langmuir profiles. Figure 17 shows Langmuir profiles taken as a function of radial distance from the dipole center for different backfill pressures. The profiles are taken shot to shot by moving the equatorial Langmuir probe out in radial distance away from the M2P2 magnet. At lower neutral backfill, the radial profile is seen to remain peaked around $35 \mathrm{~cm}$ from the dipole axis. This position corresponds to the location that source magnetic field lines map into the equatorial region. At the $10^{-5}$ Torr level peak current is reduced and the profile has slightly broadened as plasma losses are increased. Upon reaching the $10^{-4}$ Torr level, the total plasma in the equatorial region is seen to grow and the profile is now very broad. Plasma has been produced or radially diffused across the entire radial distance measured, with peak current seen at $25 \mathrm{~cm}$. A small increase in
Langmuir current is seen at the $35 \mathrm{~cm}$ location, but overall the original peaked profile is completely lost.

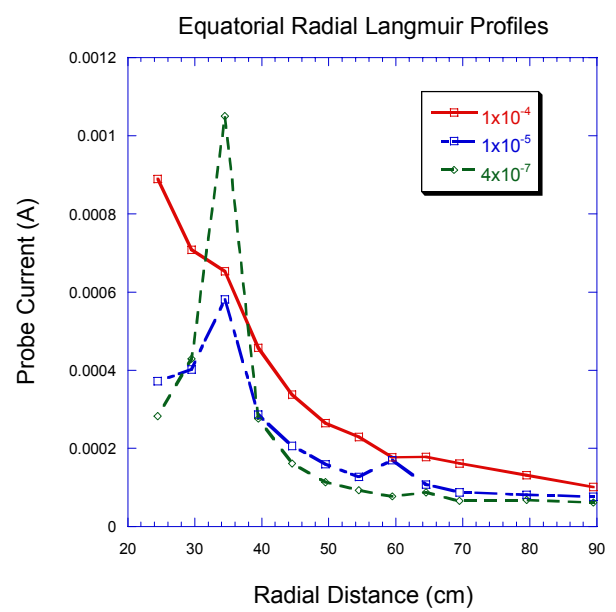

Figure 17, Radial Langmuir profiles for various neutral backfill pressures

\section{Equatorial Electron Energy Analyzer}

An electron energy analyzer was also used to measure the electron energy distribution function (EEDF) in the equatorial region. The distribution function, $f$, is directly proportional to the derivate of the characteristic current vs voltage curve as given by

$$
f\left(v_{\|}\right) \equiv \int_{-\infty}^{\infty} \int_{-\infty}^{\infty} f\left(v_{\|}, v_{\perp}\right) d v_{\perp}
$$

and

$$
j=p^{2} f
$$

Where $\mathrm{j}$ is the differential flux as measured with the analyzer. ${ }^{10}$ Here, $p$ is the momentum, with $p=m U, m$ is the electron mass and and $U$ the velocity.

Figure 18 is the electron energy distribution as a function of energy. This data is for argon in the equatorial region without neutral backfill. The distribution is peaked at $120 \mathrm{eV}$, with a much narrower width, showing a beam like distribution. To determine the overall characteristics of the distribution, fitting was attempted using a drifting maxwellian model for the distribution function. Additionally, a model, which included a secondary low energy electron population, was also conducted. The best fit is 
shown as the dashed line in Figure 18. For the case of no neutral argon back fill, the fit gives an electron temperature of $22 \mathrm{eV}$ with a nonthermal drift of $97 \mathrm{eV}$. The electron temperature of the fit is similar to temperatures found with the swept Langmuir probe for same neutral pressures. The high drift to the plasma again suggests a directional coupling down stream of the helicon coil to the bulk of the elections.

Other experiments using electron energy analyzers have not shown such high non-thermal drifts, but are usually run in much higher neutral pressures.

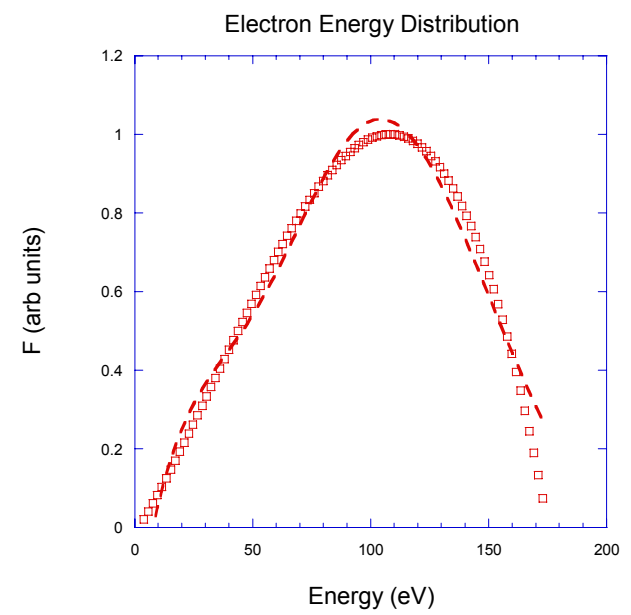

Figure 18, Electron Energy Distribution. Boxes are the reconstructed data points. Dashed line represents best fit to data.

Added collisions with neutrals will cause increased ionization and thermalize the plasma. This effect can be seen in Figure 19, which is the energy distribution with a $10^{-4}$ background neutral fill. In this case, fitting to data produces an electron temperature of $10 \mathrm{eV}$ and a thermal drift of $75 \mathrm{eV}$. Coupling to the background neutral population increases the low energy portion of the distribution and reduces its width. The peak location of the distribution has also been shifted slightly to the left corresponding to a lower drift speed. This effect actually increases the temperature in low energy population of the electron distribution, but is difficult to resolve in the distribution function due to screening of these electrons by the sheath potential around the analyzer.

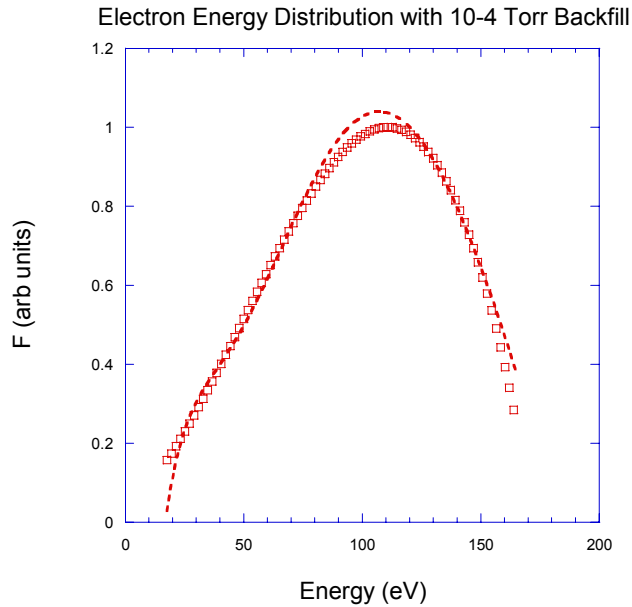

Figure 19, Electron Energy Distribution with $10^{-4}$ Torr backfill pressure. Boxes are reconstructed data points. Dashed line is best fit to data.

\section{CONCLUSIONS AND FUTURE WORK}

The M2P2 prototype and helicon source is capable of operation over a wide range of conditions, allowing determination of optimal performance parameters. The helicon source was able to run in a pulsed manner with a steep neutral pressure gradient from the source region to the equatorial region. The source could operate near $100 \%$ ionization efficiencies for optimal input gas flow rates, with peak density output at roughly $80 \%$ ionization efficiency. Data suggests that increasing helicon source power levels will lead to increased plasma production while maintaining the same ionization efficiencies.

In the dipole equatorial region density profiles were seen to follow source density profiles and density was seen to increase with time until plasma loss rates balanced plasma inflow from the source. Equatorial plasma temperatures were higher then the source region showing additional energy coupling down stream away from the helicon coil. When operated in argon, with low background neutral pressures in the equatorial region, measured electrons temperatures are higher by at least a factor of 2 then any other helicon experiment to our knowledge. This is advantageous as it allows for plasma heating away from the source area where losses are large due to the wall boundary and neutral collisions. Energy coupling down stream was also confirmed with 
the addition of neutral backfills into the chamber. As the backfill pressure was increased the electron temperature was seen to fall to levels consistent with other helicons at similar fill pressures. Plasma densities were seen to initially fall with increasing fill pressures, but then rise sharply as the $\mathrm{mfp}$ for ionization increased and secondary plasma production occurred in the equatorial region.

In summary, the helicon plasma source for the M2P2 prototype is able to operate as prescribed with plasma parameters meeting or exceeding original requirements. Down stream effects by neutrals was found to be large and can be an additional energy loss channel. Further work will concentrate on increasing source power to increase plasma pressures in the equatorial region.

\section{BIBLIOGRAPHY}

${ }^{1}$ J. Squire, F. Chang Diaz, V. Jacobson and G. McCaskill, "Helicon Plasma Injector and Ion Cyclotron Acceleration Development in the VASIMR Experiment," $36^{\text {th }}$

AIAA/ASME/SAE/ASEE Joint Propulsion Conference Proceedings, 17-19 July, 2000

2 J. Gilland, "The Potential for Compact Helicon Wave Sources for Electric Propulsion", Proc. of $27^{\text {th }}$ International Electric Propulsion

Conference, IEPC-01-210, 2001

${ }^{3}$ R. Boswell, "Very Efficient Plasma Generation By Whistler Waves Near the Lower Hybrid Frequency," Plasma Phys. Control. Fusion, 26, 1147, 1984

${ }^{4}$ D. Miljak and F. Chen, "Density limit in helicon discharges", Plasma Sources, Sci. and Technol.,7, 537, 1998

${ }^{5}$ D. Blackwell, F. Chen, "Time-resolved measurements of the electron energy distribution function in a helicon plasma", Plasma Sources, Sci. and Technol., 10, 226, 2001

${ }^{6}$ R. Winglee, T. Ziemba, P. Euripides and J. Slough, "Computer Modeling of the Laboratory Testing of Mini-Magnetospheric Plasma Propulsion (M2P2)“, International Electric
Propulsion Conference Proceedings, October 14-19, 2001

${ }^{7}$ R. Winglee, J. Slough, T. Ziemba, and A. Goodson, Mini-magnetospheric plasma propulsion: Tapping the energy of the solar wind for spacecraft propulsion, J. Geophys. Res., 105, 21,067, 2000.

${ }^{8}$ I. Sudit and F. Chen, "RF Compensated Probes for High-Density Discharges," Plasma Sources Sci and Tech, 3, 162, 1994

${ }^{9}$ G. Conway, A. Perry and R. Bowell, "Evolution of ion and electron energy distributions in pulsed helicon plasma discharges", Plasma Sources Sci and Tech, 7, 337, 1998

${ }^{10}$ G. Parks, "Physics of Space Plasmas", Addison-Wesley Publishing Company, 1991, p.38 